\title{
Learning outcomes of occupational therapy and physiotherapy students during their community-based education attachment
}

\author{
T Ndlovu, BSc, HPT; T M Chikwanha, MPH, BSc (HOT); N Munambah, MSc (OT), BSc (HOT)
}

Department of Rehabilitation, College of Health Sciences, University of Zimbabwe, Harare, Zimbabwe

Corresponding author: T M Chikwanha (middychiky@yahoo.co.uk)

Background. Community-based education (CBE) is a learning approach that enables students to acquire skills by means of applied learning in the community. The goals of CBE include creating adequate knowledge, skills and attitudes that facilitate service delivery in communities. These goals ensure the provision of efficient health services to marginalised communities with significant resource constraints. The Department of Rehabilitation at the University of Zimbabwe, Harare, has adopted CBE as one of the learning approaches that enables students to acquire comprehensive skills for future service provision in the community.

Objectives. To determine the learning outcomes of rehabilitation students at the University of Zimbabwe and to identify factors affecting learning during the CBE attachments.

Methods. A descriptive cross-sectional survey was conducted at the University of Zimbabwe. Thirty-five final-year occupational therapy and physiotherapy students responded to a self-administered questionnaire. Data were analysed using Microsoft Excel 2013 (USA) and SPSS version 21 (IBM Corp., USA).

Results. All the students reported that the CBE attachment improved their community-engagement skills, professional skills and personal growth. Barriers to effective learning included inadequate transport (100\%), poor internet connectivity (82\%), lack of research-related objectives (74\%) and inadequate resources for CBE activities (60\%).

Conclusion. The CBE attachment enabled students to gain skills in community engagement, professionalism and personal growth. However, more funding directed towards CBE activities and introducing objectives related to research, are some of the strategies that might improve the students' learning outcomes during the attachments.

Afr J Health Professions Educ 2017;9(4):189-193. DOI:10.7196/AJHPE.2017.v9i4.958

Community-based education (CBE) is a learning approach that enables students to acquire skills by means of applied learning in the community. ${ }^{[1]}$ This learning approach includes elements of primary healthcare, health promotion and disease prevention, and allows students to acquire clinical, research, communication and other professional competencies in a community setting. ${ }^{[2]}$ The goals of CBE include creating knowledge, skills and attitudes among students to ensure the provision of efficient health services to marginalised communities, often in rural areas and communities with significant resource constraints. ${ }^{[3]}$ These goals ultimately increase health professionals' willingness to work in underserved areas. ${ }^{[4]}$

CBE provides opportunities to acquire research, clinical and public health skills through applied learning in a community setting. ${ }^{[1]}$ The objective of $\mathrm{CBE}$ is to direct health professionals towards the most important health problems of the community. ${ }^{[5]}$ Despite the several challenges that may affect $\mathrm{CBE}$, lower attrition rates, a greater perceived ability to function in rural communities and high satisfaction, as indicated by students and community members, have been noted as advantages of CBE ${ }^{[6]}$ Other beneficial aspects of CBE include early contact with the community, improved teamwork of trainees, and improved interpersonal relationships and communication skills. ${ }^{[6]}$

The physiotherapy and occupational therapy students at the University of Zimbabwe, Harare, have been part of CBE attachments since the inception of the programmes in 1987. The University of Zimbabwe has 12 CBE attachment sites, which are shared by all the programmes of the College of Health Sciences. The activities at each of the CBE sites differ, depending on available resources. These activities are mainly sponsored by the Ministry of Health and Child Care and its partners. The University of Zimbabwe provides transport for the students during the CBE attachment.

Each of the attachment sites can accommodate a maximum of 10 students; therefore, the number of attachment sites per given year is determined by the number of students registered for their 3rd year in both physiotherapy and occupational therapy. The students who participated in this study had been attached to 5 of these 12 sites.

Before their CBE attachment, the students in the Department of Rehabilitation at the University of Zimbabwe receive lectures to prepare them for the attachment. Some of the content covered during the lectures includes the role of rehabilitation professionals in the community, developing, monitoring and evaluating community-based rehabilitation programmes, as well as managing and administering rehabilitation units. These lectures provide the students with the theoretical learning experience, while the $\mathrm{CBE}$ attachments provide a practical learning experience.

The students are placed at CBE attachment sites for 6 weeks in teams comprising occupational therapy and physiotherapy students. As part of the attachment, the students are expected to visit the local leadership, such as chiefs, headmen, councillors and traditional healers, before conducting any rehabilitation intervention in the community. They are also 
expected to participate in management and administration activities in the rehabilitation departments. The students are supervised by rehabilitation staff in the districts where they are attached. In addition, the faculty from the Department of Rehabilitation conduct a once-off mid-attachment evaluation for each of the sites.

For the training curriculum to remain relevant in the context of CBE, there is a need to continuously evaluate learning outcomes for students. This study was conducted to determine the learning outcomes, barriers and facilitators to effective learning of physiotherapy and occupational therapy students at the University of Zimbabwe during their CBE attachment.

\section{Methods}

A descriptive cross-sectional study was conducted in the Department of Rehabilitation, College of Health Sciences, University of Zimbabwe. The university is currently the only institution that trains physiotherapists and occupational therapists in Zimbabwe, with an average enrolment of 40 per year for both.

Guided by the objectives of the CBE attachment and insights from the literature, the researchers developed a self-administered questionnaire that was used in the study. This questionnaire had three sections. Section A collected demographic information. Section B consisted of 20 questions related to students' learning outcomes during the CBE attachment. These questions were further divided into three subsections, i.e. community engagement, professional skills and personal growth. Community engagement was defined as the cognitive, affective and behavioural outcomes related to community participation. ${ }^{[7]}$ Professional skills were described as techniques, behaviours and attitudes that enhance efficacy in the workplace. ${ }^{[7]}$ Personal growth was defined as affective outcomes related to understanding oneself. ${ }^{[7]}$ Community engagement was assessed using 6 questions, professional skills were assessed using 10 questions and personal growth was assessed using 4 questions. The responses to the questions that assessed the learning outcomes were rated on a 4-point scale: $1=$ not at all, $2=$ minimally, $3=$ moderately, and $4=$ extensively. Section $\mathrm{C}$ assessed the barriers to $\mathrm{CBE}$ and the possible strategies for improving learning experiences during the attachment. This section had closed-ended questions with 'yes' or 'no' responses.

Validation of the questionnaire was done in two stages. Firstly, the questionnaire was given to a panel of experts who included physiotherapy and occupational therapy lecturers responsible for the CBE programme. After revising the questionnaire based on the feedback from the panel of experts, a pilot study was conducted with 20 of the physiotherapy and occupational therapy students who had graduated from the University of Zimbabwe in 2015. The data from the pilot study were then analysed and redundant questions were removed.

The study sample comprised 35 occupational therapy and physiotherapy students who had just started the first semester of their final year. An overview of the study and its objectives was presented to these students before the self-administered questionnaires and consent forms were distributed. Students who were willing to participate were asked to sign the consent forms first and complete the questionnaire, which they had to drop into a box in their lecture room within 1 week. No names or student identification numbers were recorded on the questionnaires.

Data were entered into Microsoft Excel 2013 (USA) and analysed using SPSS version 21 (IBM Corp., USA). Sociodemographic characteristics were analysed for means and frequencies. Students' level of community engagement was scored out of a maximum possible score of 24 and a minimum possible score of 6. Professional skills were scored out of a maximum possible total of 40 and minimum possible score of 10. Personal growth was scored out of a maximum possible score of 16 and a minimum possible score of 4 . Total expected scores on community engagement (24), professional skills (40) and personal growth (16) were added to give the expected overall learning outcome score of 80 . The overall learning outcome score for each of the students was obtained by expressing the total scores from each of the three subsections as a percentage of the overall expected learning outcome score. Perceived barriers were grouped into three categories, i.e. administrative challenges, personal barriers and financial challenges, and were presented as frequencies. Strategies to facilitate students' learning during the $\mathrm{CBE}$ attachment were also expressed as frequencies.

\section{Ethical approval}

Permission to carry out the study was requested from the dean, College of Health Sciences, University of Zimbabwe. Ethical approval was sought from the Joint Research Ethics Committee (ref. no. JREC/253/15) and from the Medical Research Council of Zimbabwe (ref. no. MRCZ/B997).

\section{Results}

All 35 final-year students participated in the study. The mean age of participants was 22 (standard deviation (SD) 0.97) years. Twenty-three (65\%) of the participants were female and 12 (34\%) were male. Twenty-one (60\%) participants were physiotherapy students and 14 (40\%) occupational therapy students. Six (17\%) students were attached in district 1; 7 (20\%) in district 2; 8 (23\%) in district 3; 7 (20\%) in district 4; and 7 (20\%) in district 5. (Note that the actual names for the districts were replaced with numbers for confidentiality purposes.)

Students' self-reported learning outcomes of the CBE attachments are given in Table 1.

\section{Community engagement}

All students had an opportunity to learn about community engagement during their CBE attachment (Table 1). All of them reported that the CBE attachment had enhanced their understanding of community issues pertaining to rehabilitation, facilitated their understanding of clients in need of rehabilitation services, and improved their understanding of the organisation of rehabilitation services at community level. They also learnt how to conduct health education talks and provide outreach services.

\section{Professional skills development}

The students generally reported that the CBE attachment had given them an opportunity to improve their professional skills (Table 1). The majority (97\%) reported that their learning experiences had helped them to interact with other health professionals in patient care and enhanced their conflict-resolution skills. All the students reported that the attachment had improved their public speaking skills and their ability to work as part of a team. The attachment also improved the ability of 24 (68\%) students to work independently.

With regard to the development of management skills, most (91\%) of the students reported that the CBE attachment had improved their ability to run meetings, delegate tasks and respect the opinions of others when working as a team. Nineteen (54\%) of the students indicated that the CBE experience extensively improved their understanding of the roles of other rehabilitation staff. Twelve (34\%) of the participants indicated that the CBE experience extensively improved their understanding of how to manage a rehabilitation department. 


\begin{tabular}{|c|c|c|c|c|}
\hline Learning attribute & $\begin{array}{l}\text { Not at all, } \\
n(\%)\end{array}$ & $\begin{array}{l}\text { Minimally, } \\
n(\%)\end{array}$ & $\begin{array}{l}\text { Moderately, } \\
n(\%)\end{array}$ & $\begin{array}{l}\text { Extensively, } \\
n(\%)\end{array}$ \\
\hline \multicolumn{5}{|l|}{ Community engagement } \\
\hline Enhanced my understanding of community issues & $0(0)$ & $3(9)$ & $13(37)$ & $19(54)$ \\
\hline Will likely enhance my future community engagement & $2(6)$ & $2(6)$ & $13(37)$ & $18(51)$ \\
\hline Facilitated my understanding of clients in need of rehabilitation services & $1(3)$ & $2(6)$ & $13(37)$ & $19(54)$ \\
\hline Helped me to understand the organisation of rehabilitation services at community level & $0(0)$ & $5(14)$ & $15(43)$ & $15(43)$ \\
\hline Deepened my understanding of educational talks & $1(3)$ & $0(0)$ & $17(48.5)$ & $17(48.5)$ \\
\hline Enhanced my ability to conduct outreaches to the community & $0(0)$ & $1(3)$ & $14(40)$ & $20(57)$ \\
\hline \multicolumn{5}{|l|}{ Professional skills } \\
\hline Helped me to interact with other disciplines in patient care & $1(3)$ & $8(23)$ & $19(54)$ & $7(20)$ \\
\hline Improved my public speaking skills & $0(0)$ & $7(20)$ & $19(54)$ & $9(26)$ \\
\hline Improved my ability to work as part of a team & $0(0)$ & $2(6)$ & $13(37)$ & $20(57)$ \\
\hline Improved my ability to work independently & $4(12)$ & $6(17)$ & $18(51)$ & $7(20)$ \\
\hline Improved my skills with regard to conflict resolution & $1(3)$ & $8(23)$ & $16(46)$ & $10(28)$ \\
\hline Improved my ability to run meetings & $3(9)$ & $6(17)$ & $17(48)$ & $9(26)$ \\
\hline Improved my ability to delegate & $3(9)$ & $3(8)$ & $16(46)$ & $13(37)$ \\
\hline Improved my ability to listen to others & $0(0)$ & $1(3)$ & $12(34)$ & $22(63)$ \\
\hline Improved my understanding of the roles of other rehabilitation staff & $1(3)$ & $2(6)$ & $13(37)$ & $19(54)$ \\
\hline Improved my understanding of how to manage a rehabilitation department & $1(3)$ & $2(6)$ & $20(57)$ & $12(34)$ \\
\hline \multicolumn{5}{|l|}{ Personal growth } \\
\hline Helped to clarify my values & $0(0)$ & $7(20)$ & $16(46)$ & $12(34)$ \\
\hline Helped to improve my personal qualities & $1(3)$ & $2(6)$ & $19(54)$ & $13(37)$ \\
\hline Improved my ability to consider the perspectives of others & $0(0)$ & $1(3)$ & $11(31)$ & $23(66)$ \\
\hline Deepened my understanding of myself & $2(6)$ & $7(20)$ & $13(37)$ & $13(37)$ \\
\hline
\end{tabular}

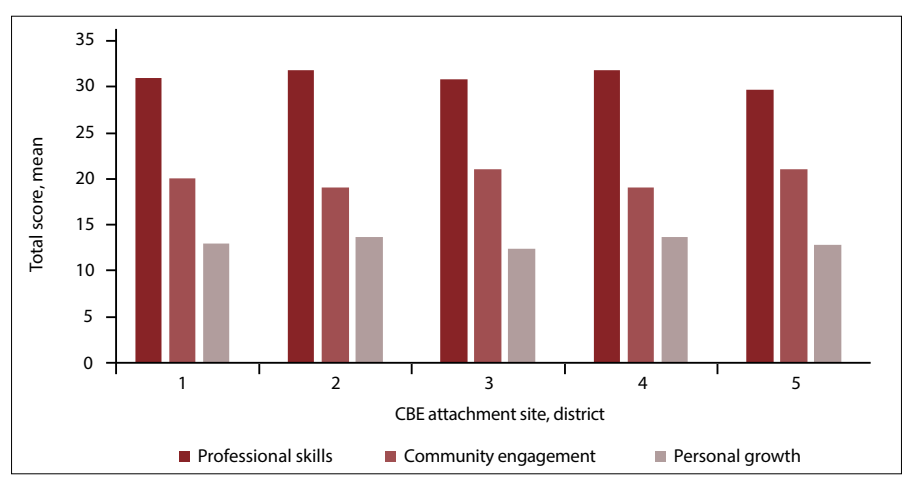

Fig. 1. Learning outcome scores by attachment site. (CBE = community-based education.)

\section{Personal growth}

All the students reported that the attachment provided an opportunity to gain skills with regard to personal growth. These included clarification of values, personal professional growth, and understanding of self and others.

\section{Learning outcome scores}

Students attached to different CBE sites presented with varying scores for community engagement, professional skills and personal growth (Fig. 1). Generally, all the students indicated the most improvement in community engagement skills and the least improvement in their personal skills.



Fig. 2. Overall learning outcome scores. (CBE = community-based education.)

The mean overall learning outcome score was 65 (SD 7.0)\%. Students placed in district 2 had the highest overall learning outcome score, while those in district 1 had the lowest score (Fig. 2).

\section{Barriers to effective CBE}

The barriers reported by students included unavailability of internet connectivity (82\%), unavailability of supervisors during some of the activities (57\%), lack of clarity in the objectives given to students (60\%) and lack of objectives related to research (74\%). Seventeen (48\%) participants identified the attachment period as being inadequate to cover all the learning 
objectives. All the students in districts 1, 2 and 3 reported a low standard of accommodation at their attachment sites. Limited access to transport to conduct CBE activities and lack of funding for CBE activities were also identified as barriers to effective learning. Failure to communicate using a local language was the only personal barrier to effective learning reported by $13(37 \%)$ participants.

\section{Proposed strategies for promoting learning}

Strategies that were proposed to improve the students' learning outcomes during CBE attachments included revising the objectives (94\%), increasing faculty involvement (97\%), providing of transport by the university throughout the attachment period (97\%), improving the state of the accommodation facilities (60\%) and introducing funds directed towards the CBE activities (19\%).

\section{Discussion}

The development of competence and confidence of students is one of the benefits of CBE experience. ${ }^{[5,8]}$ In our study, the CBE experience offered students an opportunity to improve their skills in community engagement, and develop their professional and personal skills needed to effectively provide rehabilitation services to communities.

The CBE attachment enhanced the students' understanding of clients in need of rehabilitation services. In a hospital setting, students often miss the opportunity to interact with people with disabilities in the community. ${ }^{[8]}$ CBE affords students an opportunity to interact with individuals from different backgrounds and with different abilities. ${ }^{\left[{ }^{[]}\right.}$Such interactions enable students to develop in aspects of client centredness and gain a better understanding of the clients' rehabilitation needs outside the hospital setting. ${ }^{[9]}$

In our study, the participants reported that the CBE attachment had enhanced their future engagement with the community. They also indicated improvements in their professional skills, including interpersonal skills and their ability to work as part of a team. The interaction with the community allows students an opportunity to acquire more knowledge and skills, and attitudes necessary for practice in the community. ${ }^{[6]} \mathrm{CBE}$ therefore positively impacts on the students' understanding of community issues, as it complements classroom learning. ${ }^{[10]}$

The CBE experience equipped the students with skills needed to educate the community on health-related issues, enhanced their ability to conduct outreaches in the community, and improved their public speaking skills. Several authors have reported that CBE offers students a chance to acquire skills in public health services and health promotion. ${ }^{[1-3,5]}$ Such exposure affords students an opportunity to learn about health services within the community.

Generally, all students improved in professional skills, including interpersonal skills and their ability to work as part of a team. Interprofessional learning helps students to gain the skills necessary to promote teamwork, communication and collaborative learning. ${ }^{[3,11]}$ Such interactions provide them with opportunities to interact with a multidisciplinary team and, as such, they will have an appreciation of the roles of each of the team members

The students also reported that the attachment had improved their ability to work independently, as well as their skills with regard to conflict resolution. Practice placements, such as CBE, develop students' self-confidence, thereby facilitating their ability to work independently. Such learning experiences also develop their professional skills, including conflict management. ${ }^{[6,9]}$ From the researchers' personal experience, for students to fulfil the CBE attachment objectives, they have to work together as a team. Conflicts usually arise when tasks have to be shared and they do not agree on how it should be done. Conflict resolution skills will then develop, as students learn to negotiate and agree on how tasks should be shared within the team.

Several authors have identified the unavailability of supervisors during some of the CBE activities as a challenge hindering effective learning during attachments. ${ }^{[3,4,6,12]}$ As noted in the current study, despite all occupational therapy and physiotherapy students being attached at district level, there was a variation in overall learning outcome scores based on different attachment sites. This indicates that students had different learning experiences based on the site to which they were attached. Therefore, there is a need to train supervisors and to agree on a model of providing supervision and support services to the students. The introduction of an efficient internet service that facilitates access to e-learning resources may also assist in overcoming the issue of inadequate supervision. To promote effective CBE programmes, e-learning is important, as it permits real-time communication between faculty and students during their attachment, which can help in the daily supervision of students. ${ }^{[4]}$

Unavailability of transport was reported to be a major barrier to effective learning, possibly resulting in clients living in very remote areas not receiving the necessary community rehabilitation services. Additional resources, including transport, are essential in facilitating the effectiveness of CBE programmes. ${ }^{[2,13]}$ Identifying alternative ways of ensuring that transport is available throughout the duration of the placement and introducing funds directed towards $\mathrm{CBE}$, are strategies that can be employed to address transport problems in order to improve students' learning outcomes.

Inadequate time and lack of clear objectives related to research were also reported as barriers to effective learning. The tendency to underestimate the amount of time needed by students for their CBE attachments has been linked to lack of clear objectives in various settings. ${ }^{[3,5,14]}$ Having clear and specific learning objectives enables the students to know exactly what is expected of them; ${ }^{[4,13]}$ therefore, they are able to allocate adequate time for all their CBE activities. Periodically revising the CBE objectives may assist in ensuring that these remain feasible and contextually relevant.

Low-standard accommodation was perceived as a barrier to effective learning by the physiotherapy and occupational therapy students who participated in this study. Unavailability of internet access, poor water and electricity supplies, outdated room appliances and poor security are some of the issues that negatively contribute to accommodation standards. ${ }^{[3,4]}$ Currently, the maintenance for most of the hostels used by students during the CBE attachment is the responsibility of the hospitals where these hostels are built. With the prevailing economic hardships in Zimbabwe and the recent freezing of posts by the Ministry of Health and Child Care, most hospitals have shortages of cleaning staff. Cleaning of the students' accommodation may not even be on the list of cleaning priorities for the hospitals.

\section{Study limitations}

This study was conducted to determine the learning outcomes of rehabilitation students at the University of Zimbabwe and to identify factors affecting learning during the CBE attachments. It would have been ideal to establish the consistency of the study instrument before conducting the research. However, the data collection instrument did not undergo reliability testing, and the authors acknowledge this omission as a limitation of the study. 


\section{Conclusion}

Experiential learning during the CBE attachment for occupational therapy and physiotherapy students offered them an opportunity to apply theoretical knowledge to practise and develop personal, professional and community engagement skills. However, barriers, including lack of clear research-related objectives, limited resources for CBE activities, inadequate supervision and limited exposure to communities in terms of time allocated for the attachment, negatively affected the learning experiences. The availability of more resources for $\mathrm{CBE}$ activities may improve the overall learning experiences of students.

Acknowledgements. Our most sincere gratitude is extended to lecturers at the Department of Rehabilitation, College of Health Sciences, University of Zimbabwe, for their assistance and guidance throughout the research project; the Joint Research Ethics Committee of the College of Health Sciences; the Medical Research Council of Zimbabwe for reviewing the study protocol; the dean of the College of Health Sciences for granting permission to carry out this study; to the physiotherapy and occupational therapy students at the University of Zimbabwe, who participated in the study; and to the physiotherapists and occupational therapists who participated in the pilot study.

Author contributions. TN: developed the research protocol, collected and analysed the data, and contributed to writing the manuscript; TMC: developed the research protocol, collected and analysed the data, and contributed to writing the manuscript; NM: analysed the data and contributed to writing the manuscript.

Funding. None.

Conflicts of interest. None.
1. Dreyer A, Couper I, Bailey R, Talib Z, Ross H, Sagay A. Identifying approaches and tools for evaluating community-based medical education programmes in Africa. Afr J Health Professions Educ 2015;7(1):134-139. https://doi.org/10.7196/AJHPE.568

2. Bailey RJ, Baingana RK, Couper ID, et al. Evaluating community-based medical education programmes in Africa: A workshop report. Afr J Health Professions Educ 2015;7(1 Suppl 1):140-144. https://doi.org/10.7196/ AJHPE. 475

3. Kaye D, Mwanika A, Burnham G, et al. The organization and implementation of community-based education programs for health worker training institutions in Uganda. BMC Int Health Hum 2011;11(1):1. https://doi. org/10.1186/1472-698X-11-S1-S4

4. Mariam DH, Sagay AS, Arubaku W, et al. Community-based education programs in Africa: Faculty experience within the Medical Education Partnership Initiative (MEPI) network. Acad Med 2014;89(8):S50-S54. https://doi. org/10.1097/ACM.0000000000000330

5. Kaye DK, Muhwezi WW, Kasozi AN, et al. Lessons learnt from comprehensive evaluation of community-based education in Uganda: A proposal for an ideal model community-based education for health professional training institutions. BMC Med 2011;11(1):1. https://doi.org/10.1186/1472-6920-11-7

6. Wald HS, Davis SW, Reis SP, Monroe AD, Borkan JM. Reflecting on reflections: Enhancement of medical education curriculum with structured field notes and guided feedback. Acad Med 2009;84(7):830-837. https: $/ /$ doi.org/10.1097/ACM.0b013e3181a8592

7. Lichtenstein G, Tombari M, Thorme T, Cutforth N. Development of a national survey to assess student learning outcomes of community-based research. J High Educ Outreach Engage 2011;15(2):7-34.

8. Shields N, Taylor NF. Physiotherapy students' self-reported assessment of professional behaviours and skills while working with young people with disability. Disabil Rehabil 2014:36(21):1834-1839. https://doi.org/10.3109/096 38288.2013 .871355

9. Yorio PL, Ye F. A meta-analysis on the effects of service-learning on the social, personal, and cognitive outcomes of learning. Acad Manag Learn Educ 2012;11(1):9-27. https://doi.org/10.5465/amle.2010.0072

10. Davies K. Harrison K Clouder D, Gilchrist M, McFarland I Earland J. Making the transition from physiotherapy student to interprofessional team member. Physiotherapy 2011;97(2):139-144. https://doi. org/10.1016/j.physio.2010.08.00

11. Rodger S, Fitzgerald C, Davila W, Millar F, Allison H. What makes a quality occupational therapy practice placement? Students' and practice educators' perspectives. Aust Occup Ther J 2011;58(3):195-202. https://doi org/10.1111/j.1440-1630.2010.00903.x

12. Burggraaf A, Bourke-Taylor H. Occupational therapy students' fieldwork placement: Institutional an community based rehabilitation models in the Solomon Islands. N Z Occup Ther J 2008;55(2):25.

13. Okayama M, Kajii E. Does community-based education increase students' motivation to practice community health care? A cross sectional study. BMC Med 2011;11(1):1. https://doi.org/10.1186/1472-6920-11-19

14. Kristina TN, Majoor GD, van der Vleuten CP. Defining generic objectives for community-based education in undergraduate medical programmes. Med Educ 2004;38(5):510-521. https://doi.org/10.1046/j.1365-2929.2004.01819

Accepted 23 May 2017 